\title{
Successful Management of a Stab Wound to the Left Ventricle
}

\author{
Jaleed Ahmad Gillani ${ }^{1}$, Hamza AR Khan ${ }^{2}$, Omar Irfan², Saulat H Fatimi ${ }^{3}$ \\ ${ }^{1}$ Medical College, Ziauddin University, Karachi, ${ }^{2}$ Medical College, Aga Khan University, Karachi, \\ ${ }^{3}$ Department of Surgery, Division of Cardiothoracic Surgery, Aga Khan University, Karachi
}

Key Words : tab wound, Left ventricle, Cardiac tamponade.

\begin{abstract}
:
A 24 year old male presented to the emergency room with a 2 hour history of knife stab wound. He was haemodynamically stable with good bilateral air entry. However on X-ray an enlarged cardiac silhouette was seen. The patient suddenly became hypotensive and on suspicion of cardiac tamponade a catheter was introduced in pericardial space, but the measure was unsuccessful and he was rushed to the Operating Room. During the surgery a $2 \mathrm{~cm}$ long laceration was identified in the mid-left ventricular ( $L V)$ wall less than $1 \mathrm{~mm}$ away from the Left Anterior Descending (LAD) Artery. The Left Anterior Descending Vein was damaged which was then repaired along with the ventricular wall by the interrupted pledgeted 3-0 prolene suture, whilst a cardiopulmonary bypass was kept on the stand by. The patient was checked for a ventricular septal defect via an intraoperative echocardiography. Subsequently, he had an uneventful recovery and was discharged.
\end{abstract}

(Cardiovasc.j. 2016; 9(1): 60-63)

\section{Introduction:}

Gunshot and stab wounds are the commonest causes of cardiac penetrating trauma. ${ }^{1}$ However, the phenomenon of cardiac tamponade following stab wounds is infrequent, with approximately $2 \%$ of penetrating injuries and $10 \%$ of blunt trauma injuries being reported to result in cardiac tamponade. ${ }^{2}$ Compared to the right ventricle, left ventricular penetrating trauma cases are relatively rarer due to its postero-lateral position. ${ }^{3}$ Stab wounds to the heart can serve to be fatal and in Karachi, $7.6 \%$ of deaths from stab wounds involved the heart. ${ }^{4}$ In this case the site of injury was the uncommonly affected left ventricle being in very close proximity (less than $1 \mathrm{~mm}$ ) to the LAD, damage to which is associated with a mortality of $89 \%$ and requires use of a cardiopulmonary bypass during surgical management. ${ }^{5}$ Likewise, within Pakistan, even though there has been a case report of cardiac tamponade developing after gunshot wounds, there is no case report on cardiac tamponade following stab wounds and we chose to present this case report to document our experience of managing this rare finding.

\section{Case Presentation:}

A 24 year old male presented to the emergency room with a history of a knife stab wound located in the left $4^{\text {th }}$ intercostal space in the midclavicular line which he suffered two hours back. At the time of presentation he was hemodynamically stable with a heart rate of 110 beats per minute, Blood pressure 110/80 mmHg, Respiratory Rate 26 breaths per minute along with good air entry bilaterally.

Suddenly the patient became hypotensive and drowsy. So, on the suspicion of cardiac tamponade, a catheter was inserted in the sub-xiphoid area for its relief but that measure was unsuccessful. Chest radiograph was performed showing an enlarged cardiac silhouette however it showed no hemo pneumothorax.

The patient was rushed to the operating room for an emergency median sternotomy. The cardiac tamponade was identified and relieved by opening the pericardium longitudinally. There was a peroperative finding of a $2 \mathrm{~cm}$ long laceration in the mid-LV wall less than $1 \mathrm{~mm}$ from the LAD (Fig.1). The artery was not injured but the Left Anterior Descending vein was damaged.

The Ventricular wall and the injured Left Anterior Descending vein were repaired with the interrupted pledgeted 3-0 prolene suture, (Fig.2) with a cardiopulmonary bypass kept on standby. The artery was handled carefully to prevent any injury as it was lying in close proximity of the

Address of Correspondence: Dr. Omar Irfan, Medical College, Aga Khan University, Karachi, Pakistan. Emailomarirfan1@hotmail.com 


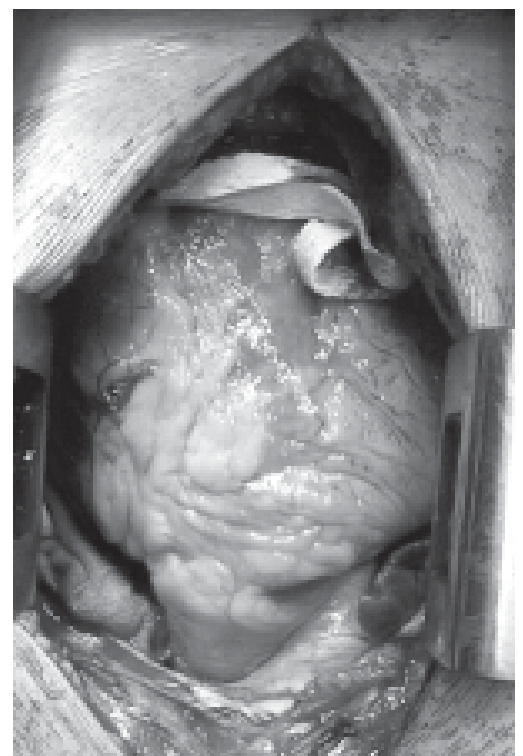

Fig.-1: $2 \mathrm{~cm}$ long laceration in the mid left ventricular wall less than $1 \mathrm{~mm}$ from the left anterior descending artery as viewed from the cranial end.

injured vein. The possibility of ventricular septal defect was excluded by doing an intra-operative echo. The patient had an uneventful recovery course, and was discharged home in perfect health with no residual damage. He was followed up for a month and had no active symptoms.

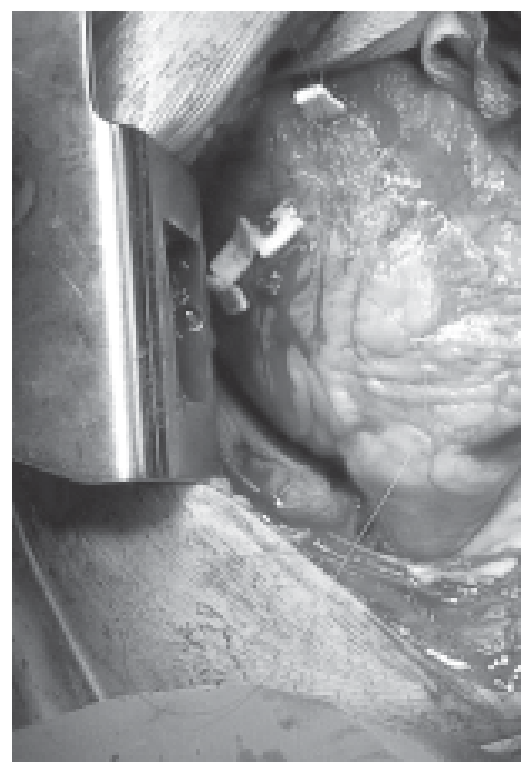

Fig.-2: Laceration in the mid left ventricular wall repaired with the interrupted pledgeted 3 - 0 prolene suture.

\section{Discussion:}

In comparison to the gunshot wounds, stab wounds serve to be less lethal and occur in lesser frequency. ${ }^{5}$ In Karachi, most of the victims of the death from stab wounds tend to be male, being four times greater in number than females. Likewise, $59.3 \%$ of victims of stab wounds are young people belonging to the age group of 20-39 years. $^{4}$

Cardiac Tamponade is defined as a life-threatening, slow or rapid compression of the heart due to the accumulation of pericardial fluid, pus, blood, clots, or gas, as a result of effusion, trauma, or rupture of the heart. ${ }^{7}$ Cardiac tamponade may be of a wide variety of causes which can be broadly classified into haemorrhagic, serous or sero-sanguinous and purulent. Haemorrhagic causes, relevant to our case, include a sudden accumulation blood in pericardial space from a breached myocardium following myocardial infarction or penetrating cardiac trauma. ${ }^{8}$

Penetrating cardiac injuries are greater in frequency at some sites due to their anatomical position. The right ventricle is the most frequently affected site due to its ventral anatomical position, followed by the left ventricle, right atrium and left atrium. ${ }^{3}$

Patients with acute cardiac tamponade generally present with a triad of symptoms, the Beck's triad (distended neck veins, hypotension and muffled heart sounds). ${ }^{9}$ Other symptoms such as dyspnea, fatigue and light-headedness serve to establish a diagnosis of the condition, as was seen in our case where the patient developed hypotension and drowsiness. ${ }^{8}$

It should be understood, however, that a patient's appearance at presentation does not provide or predict the severity of the underlying injury and hence it is vital to use diagnostic modalities such as the focused assessment with sonography for trauma (FAST) to indicate free pericardial and intraperitoneal fluid in a severely injured patient during early management. ${ }^{10}$

The chest X-ray maybe within normal parameters in a case of acute cardiac tamponade however suggestive features of the condition include an enlarged cardiac shadow. ${ }^{8}$ This was what was seen in our case. Though an enlarged cardiac silhouette 
in a patient with clear lung fields can indicate the presence of pericardial effusion, chest films per se may not be helpful in initial assessment as at least $200 \mathrm{ml}$ of fluid must accumulate before the cardiac shadow is affected, reducing the efficacy of radiography. ${ }^{7}$

Echocardiography is used in establishing a diagnosis of pericardial effusion and cardiac tamponade and is superior to conventional radiography in detecting the condition by being noninvasive, definitive and sensitive enough to detect as small a volume as $20-50 \mathrm{ml}$ of pericardial fluid. 7,11 Absence of effusion generally rules out cardiac tamponade. It has also been reported that absence of collapse of cardiac chambers on echocardiography has good correlation with absence of tamponade. ${ }^{11}$ Echocardiography has many other roles as well such as detecting intrapericardial adhesions and clots as well as assessing whether pericardiocentesis is suitable or not in the case and in monitoring the procedure. $^{12}$

Cardiac tamponade is a medical emergency and for clinically unstable patients, a rapid pericardiocentesis or surgical evacuation of pericardial fluid is obligatory. ${ }^{2}$ Percutaneous balloon pericardiotomy can also be performed as an alternative to treat cardiac tamponade. ${ }^{2}$ Pericardiocentesis is classically done via 3 approaches: The Subcostal (subxiphoid) is reserved for emergencies and is probably the safest for a blind approach. The Parasternal approach is from the left sternal boundary. The Apical approach is probably the safest in terms of reduced cardiac complications, but due to its proximity to the pleural space, it carries an increased risk of pneumothorax. $^{2}$

Pericardiocentesis under local anesthesia is usually performed for the early management of patients with cardiac tamponade developing hypotension, as in our case. ${ }^{2}$ Surgical Pericardiotomy is carried out in patients presenting with acute traumatic hemopericardium like our patient but it is also indicated in compartmentalized effusion and pericardiac hematoma.$^{2}$

Median sternotomy performed in this case is a surgical procedure providing the best access to the heart whilst carrying out surgical pericardiotomy. ${ }^{1}$ Ideally, pledgetted sutures are used in order to repair Ventricular wounds so that the sutures do not cut through the myocardium. Teflon felt or autologous pericardium can be used as pledgets. ${ }^{1}$ Caution should be taken whilst suturing wounds in close proximity to coronary vessels in order to prevent them from being damaged during the procedure.

Injuries to the coronary arteries subsequent to penetrating cardiac trauma are rare. The most common injured artery is the LAD, and its vulnerability stems from its anterior location. A lot of cases presenting with injuries to the coronary arteries are young men, being a victim of violent social altercation. ${ }^{13}$

Acute cardiopulmonary bypass is generally reserved for proximal coronary artery damage along with intractable cardiac failure. The Left Anterior descending artery, due to its accessible site, is the chief coronary artery associated with significant cardiac dysfunction when injured with Asensio and his colleagues reporting a mortality rate of $89 \%$ associated with coronary artery injury. ${ }^{5,13}$ It is for these reasons juxta LAD artery and LV injuries are difficult to manage and may require cardiopulmonary bypass and grafts to $\mathrm{LAD}$ but in this case the injury was managed without going on the cardiopulmonary bypass.

Ventricular septal defect reported to occur in 1.5\%$5.0 \%$ of all penetrating cardiac traumas, did not occur in our patient which was checked by the echocardiogram, the mainstay in the diagnostic management of patients with cardiac trauma. ${ }^{14}$ Sugiyama and his colleagues and Ito and his associates have reported a case on ventricular septal defect following trauma respectively. ${ }^{14}$ Sugiyama's case report indicated use of surgery to correct the traumatic ventricular septal defect whilst in Ito's case report the patient was managed conservatively. Our patient however made an uneventful recovery in this context.

In conclusion, we have presented a case of acute cardiac tamponade occurring in a patient with penetrating trauma to the left ventricle, a site which is infrequently affected by trauma as compared to the more commonly affected right ventricle due to 
its posterolateral anatomical position. In this case, use of the acute cardiopulmonary bypass was avoided in spite of the area surgically operated on being a sensitive one.

\section{Conflict of Interest - None.}

\section{References:}

1. Kang N, Hsee L, Rizoli S, Alison P. Penetrating cardiac injury: overcoming the limits set by Nature. Injury 2009;40(9):919-927.

2. Grecu L. Cardiac tamponade. Int Anesthesiol Clin 2012;50(2):59-77.

3. Asensio JA, Berne JD, Demetriades D, Chan L, Murray J, Falabella A, et al. One hundred five penetrating cardiac injuries: a 2 -year prospective evaluation. $J$ Trauma 1998;44(6):1073-1082.

4. Mirza FH, Hasan Q, Memon AA, Adil SE-e-R. Audit of sharp weapon deaths in metropolis of Karachi-an autopsy based study. J Ayub Med Coll Abbottabad 2010;22(4):176.

5. Asensio JA, Murray J, Demetriades D, Berne J, Cornwell E, Velmahos G, et al. Penetrating cardiac injuries: a prospective study of variables predicting outcomes. J Am Coll Surgeons 1998;186(1):24-34.

6. Abbas S, Riaz MN, Zameer M, Khan AA, Ahmad W. Cardiac trauma with gunshot injuries. Journal of the
College of Physicians and Surgeons-Pakistan : JCPSP 2004;14(1):41-42.

7. Spodick DH. Acute cardiac tamponade. New Engl J Med 2003;349(7):684-90.

8. Collins D. Aetiology and management of acute cardiac tamponade. 2004.

9. Sternbach G. Claude Beck: cardiac compression triads. J Emerg Med 1988;6(5):417-419.

10. Fitzgerald M, Spencer J, Johnson F, Marasco S, Atkin C, Kossmann T. Definitive management of acute cardiac tamponade secondary to blunt trauma. Emergency Medicine Australasia 2005;17(5 6):494-499.

11. Hamid M, Khan M, Bashour AC. Diagnostic value of chest X-ray and echocardiography for cardiac tamponade in post cardiac surgery patients. JournalPakistan Medical Association 2006;56(3):104.

12. Chandraratna PA, Mohar DS, Sidarous PF. Role of echocardiography in the treatment of cardiac tamponade. Echocardiography 2014;31(7):899-910.

13. Karin E, Greenberg R, Avital S, Aladgem D, Kluger Y. The management of stab wounds to the heart with laceration of the left anterior descending coronary artery. European Journal of Emergency Medicine 2001;8(4):321-323.

14. Sugiyama G, Lau C, Tak V, Lee DC, Burack J. Traumatic ventricular septal defect. The Annals of thoracic surgery 2011;91(3):908-910. 